\title{
Realistic structuralism's identity crisis: A hybrid solution
}

\author{
Tim ButTon
}

This paper is published by Blackwell Publishing, on behalf of the Analysis Trust. This is a post-print; that is, it is the full and correct text. The definitive version is available at Blackwell. The full citation is:

Button, Tim. 2006. 'Realistic structuralism's identity crisis: A hybrid solution'. Analysis 66.3, pp.216-22.

\begin{abstract}
Keränen (2001) raises an argument against realistic (ante rem) structuralism: where a mathematical structure has a non-trivial automorphism, distinct indiscernible positions within the structure cannot be shown to be non-identical using only the properties and relations of that structure. Ladyman (2005) responds by allowing our identity criterion to include 'irreflexive two-place relations'. I note that this does not solve the problem for structures with indistinguishable positions, i.e. positions that have all the same properties as each other and exactly the same relations to all objects (including themselves). I conclude that realistic structuralists must compromise and treat some structures eliminativistically.
\end{abstract}

In this paper, I consider a specific challenge faced by realistic structuralism. I argue that neither Parsons (2004), Ladyman (2005) nor Shapiro (forthcoming) have adequately answered the problem. I advocate a hybrid position, which is realistic at its core and eliminativistic at its limits.

\section{The Automorphism Problem}

The central idea of structuralism is that systems of objects instantiate mathematical structures: each object within the system instantiates a position within the structure. Realistic structuralists claim that these structures and their positions are themselves genuine objects. So realistic structuralists believe that, for any positions within a structure (not merely for objects within systems) there is an objective fact concerning whether the positions are identical with each other 1

Realistic structuralists must provide an identity criterion to explicate the nature of such identity facts. Keränen (2001: 316) suggests that realistic structuralists must adopt:

(A) $\forall x \forall y(x=y \equiv \forall X(X(x) \equiv X(y)))^{2}$

\footnotetext{
${ }^{1}$ Cf. Keränen 2001. 328.

${ }^{2}$ Actually, Keränen offers $\forall x \forall y(x=y \equiv \forall \varphi(\varphi \in \Phi \supset(\varphi(x) \equiv \varphi(y))))$. This treats the identity of sets (e.g. $\Phi)$ as already understood, but sets are mathematical objects themselves and so should be treated by the same identity criterion. I avoid this by avoiding mention of sets.
} 
First-order variables range over positions in the structure in question. Second-order variables range over intrastructural properties, where an intrastructural property is one which mathematically characterises a position's place in its structure. Furthermore, such properties must in principle be specifiable without naming any positions within the structure; for if positions cannot by individuated by their properties, they cannot be determinately named ${ }^{3}$

Burgess (1999) and Keränen (2001) have noted that certain structures require distinct positions with identical structural properties and relations, but that this is prohibited by (A). For example:

$\mathbf{Z}+$ : the additive integers. Additive inverses have all the same intrastructural properties, so they are identified by (A).

CF: the complex field. Complex conjugates have all the same intrastructural properties, so they are identified by (A).

EP: the Euclidean plane. All points have the same intrastructural properties, so all points are identified by (A).

These examples suggest that realistic structuralism cannot countenance structures which have nontrivial automorphisms ${ }^{4}$ This is the Automorphism Problem for realistic structuralism.

Parsons has responded to the problem by challenging Keränen's 'implicit demand' that 'objects must be "individuated" by something essential or intrinsic' (2004 71). He suggests that ' $\forall X$ ' in (A) should additionally range over the relations a structure has to the systems instantiating it. Even if this is legitimate, it will not solve the Automorphism Problem. Consider a system, $S$, which instantiates $\mathbf{Z}+$. Let $a$ be the object in $S$ which instantiates the position $n_{\mathbf{Z}+}$, and $b$ be the object in $S$ which instantiates $-n_{\mathbf{Z}_{+}}$. The problem is that, since there is no purely structural difference between $n_{\mathbf{Z}+}$ and $-n_{\mathbf{Z}+}, S$ must instantiate $\mathbf{Z}+$ in two ways if it instantiates $\mathbf{Z}+$ at all; in that second instantiation, $a$ instantiates $-n_{\mathbf{Z}+}$ and $b$ instantiates $n_{\mathbf{Z}+}$. Thus $n_{\mathbf{Z}+}$ and $-n_{\mathbf{Z}+}$ have all the same relations to any systems instantiating $\mathbf{Z}+$. The Automorphism Problem cannot be solved by appealing to the relations between structures

\section{Ladyman and indiscernibles}

Realistic structuralists are free to respond by simply rejecting (A). (A) tells us that $x$ and $y$ are identical iff $x$ and $y$ have all the same monadic properties (of a restricted type). That is just the Identity of Indiscernibles and I can only direct the reader to Black 1952 as a refutation of that dubious position.

A new identity criterion is therefore required. A plausible candidate would be to individuate objects not merely by their properties but also by their relations. That

\footnotetext{
${ }^{3}$ Cf. Black 1952: 156-7.

${ }^{4} \mathrm{An}$ automorphism is an isomorphism which maps every position, relation and function of $\mathbf{S}$ onto a position, relation or function (respectively) of $\mathbf{S}$.
} 
is, $x$ and $y$ are identical iff they have all the same intrastructural relations to any object $z$ :

(B) $\forall x \forall y(x=y \equiv \forall Y \forall z(Y(x, z) \equiv Y(y, z)))$

As Ladyman (2005: 220) notes, structurally indiscernible positions hold 'irreflexive two-place relation $[\mathrm{s}]$ ' to each other. For example, $n_{\mathbf{Z}+}$ is $-n_{\mathbf{Z}+}$ 's, but not its own, additive inverse. Likewise, $a+b i_{\mathbf{C F}}$ is $a-b i_{\mathbf{C F}}$ 's, but not its own, complex conjugate. Similarly, any point on EP is at a distance from any distinct point, but it is at no distance from itself. Unlike (A), (B) is sensitive to these irreflexive relations. For example, where ' $G$ ' denotes the relation ' $x$ is the additive inverse of $y$ ', $G\left(n_{\mathbf{Z}_{+}},-n_{\mathbf{Z}+}\right)$ and $\neg G\left(n_{\mathbf{Z}+},-n_{\mathbf{Z}+}\right)$ hence, by $(\mathrm{B}), n_{\mathbf{Z}+} \neq-n_{\mathbf{Z}+}$. So the Automorphism Problem is solved for structures which require distinct indiscernibles, i.e. objects that possess all the same properties as each other, but which are distinguishable by their relations.

\section{Indistinguishables strike back}

Regrettably, certain structures also require distinct indistinguishables, where indistinguishables are objects possessing all the same properties as each other and with exactly the same relations to all objects (themselves included). (B) prohibits the existence of distinct indistinguishables. Consider a graph, G1 ${ }^{5}$

$$
b \longleftarrow a \longrightarrow c
$$

Any intrastructural relation in $\mathbf{G} \mathbf{1}$ is held either by all of $(b, c),(c, b),(b, b)$ and $(c, c)$ or by none, so (B) wrongly tells us that $b=c$. Consider also G2:

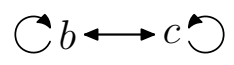

Again there is an Automorphism Problem, since every relation $b$ has to $c$, it has to itself (and vice versa). Parallel problems arise outside graph theory. Semigroups that are not groups are not closed under their (associative) binary operator, so they can (in principle) contain multiple positions that have neither any structural properties nor any structural relations to other positions. Likewise, Shapiro often discusses the finite cardinal structures, i.e. the structures consisting of a finite number of positions with no particular intrastructural properties nor relations between them. Plainly, the positions of such structures would have to be distinct indistinguishables.

We cannot solve these automorphism problems by replacing (B) with some better identity criterion. When there are indistinguishable positions, it is clear that no intrastructural properties or relations will yield the distinctness of positions, whatever identity criterion we supply.

\footnotetext{
${ }^{5}$ Keränen (2001. 321) provides a similar graph, posing the same problem for (A).
} 


\section{No primitive identity facts}

Realistic structuralists might respond by contesting the need to supply an identity criterion at all. This is Shapiro's strategy (MSb, MSa). He will argue that it is just as dubious to prohibit indistinguishables (as (B) does) as it was to prohibit indiscernibles (as (A) does). I contest the comparison. It is philosophically commonplace to accept indiscernibles; by contrast, accepting indistinguishables has several undesirable consequences.

First: accepting indistinguishables requires that identity facts are primitive, for there are no properties or relations upon which the distinctness of individuals could supervene. In addition to the obvious undesirability of postulating a new type of fact, this generates serious epistemological problems. Suppose that $m$ and $n$ have at all times all the same properties (including being at the same location, if they are located at all) and all the same relations to everything. I say that $m=n$, as does (B). Shapiro must say that I am not entitled to make this claim until I know whether $m$ and $n$ are primitively identical. But it is unclear how we could have access to primitive identity facts, and it is imperative that Shapiro address this problem, for if we cannot know whether $m$ and $n$ are one object or two, we also cannot know whether $m$ is one object, or three, or four....

Second: accepting indistinguishables commits one to an unusual notion of objecthood. The Automorphism Problem arises in $\mathbf{G} \mathbf{1}$ because $b_{\mathbf{G} \mathbf{1}}$ and $c_{\mathbf{G} \mathbf{1}}$ bear no relations to each other even though they are objects in the same configuration. This is arguably inconceivable. Such objects could not be spatial, for distinct spatial objects are invariably at a distance from each other but not from themselves (i.e. an irreflexive relation obtains between them) ${ }^{6}$ Analogies with conventional number systems likewise fail, since some irreflexive relation always obtains between distinct numbers. So if $b_{\mathbf{G} \mathbf{1}}$ and $c_{\mathbf{G} \mathbf{1}}$ are objects, they are unlike any objects with which I am familiar. Worse still are the 'objects' of some proper semigroups: what is an object with no properties and no relations? Whilst not logically incoherent, there is something metaphysically suspicious about such indistinguishables. At the very least, indistinguishables can be countenanced only if we have weaker criteria for objecthood than is usual. Shapiro must supply and defend such criteria.

These two problems show that countenancing indistinguishables requires some major metaphysical upheaval. Clearly it would be preferable, if possible, to solve the Automorphism Problem without appealing to indistinguishables.

\section{A hybrid solution}

The best response to the Automorphism Problem is to adopt a proposal which Keränen (2001: 328, n.27) and Parsons (2004: 69) both considered and rejected. The result is a hybrid version of structuralism which does not require indistinguishables.

The central idea is to distinguish between basic and constructed structures. A basic structure, $\mathbf{B}$, is treated realistically: B's positions are mathematical objects.

\footnotetext{
${ }^{6}$ Cf. Black 1952 ; Ladyman 2005.
} 
A constructed structure, $\mathbf{C}$, is treated eliminativistically as follows: talk about $\mathbf{C}$ is read paraphrastically as universal generalizations over the systems of positions in $\mathbf{B}$ which exemplify the structure $\mathbf{C}$. (This is what it means to 'construct $\mathbf{C}$ from B'.) Plainly, the 'positions' of constructed structures are not objects at all; a fortiori, there are no problems concerning intrastructural identities on constructed structures. We could avoid the Automorphism Problem by treating all problematic structures as constructed.

Parsons fears that this 'would concede a lot to Keränen' (2004: 69). If (A) were our identity criterion, it would indeed concede too much. It would be a crushing blow to realistic structuralism if it could not treat $\mathbf{Z}+, \mathbf{C F}$ and $\mathbf{E P}$ realistically. But once we have embraced (B), we can reify these structures without difficulty. Indeed, the majority of mathematical practice can be handled realistically: number theory, real and functional analysis, set theory and geometry all require only basic structures. Only those structures which would require indistinguishable positions need be treated as constructed $\sqrt{7}$ This is not too large a concession to Keränen. Where realism breaks down, it is often arguable that mathematical practice is adequately respected. For example, mathematicians do not talk of the graph $\mathbf{G 1}$ in the same way that they talk of the additive integers. I hazard that the same is true of most other structures which would require distinct indistinguishables if treated realistically ${ }^{8}$

The principle advantage of this hybrid theory is that it is immune to the main arguments raised against eliminativist structuralism. The chief problem for non-modal eliminativist structuralism is that its ontological base (namely, all actual concrete objects) is too narrow to accommodate transfinite structures. Modal structuralists attempt to avoid this problem by invoking possible objects, but the notion of 'possibility' involved is extremely obscure? These problems do not arise for the hybrid theory I propose. The (real) positions of (real) basic structures guarantee a sufficiently wide ontological base for my constructed structures. This follows from the fact that, for any well-defined structure, $\mathbf{S}$, that cannot be treated realistically, we can always define a basic structure from which $\mathbf{S}$ can be constructed. One easy way to do this is to define a structure, $\mathbf{T}$, exactly like $\mathbf{S}$ except that all its positions are well-ordered by some linear-ordering relation. $\mathbf{S}$ can be constructed from $\mathbf{T}$ and, since an irreflexive relation obtains between any pair of positions in $\mathbf{T}, \mathbf{T}$ can be treated as a basic structure. In this manner, I can always provide a basic structure from which to construct any structure that would otherwise have been problematic, so long as I am willing to postulate the basic structure. But there is no particular reason to worry about ontological parsimony; prior to discovery of the au-

\footnotetext{
${ }^{7}$ Though we are of course free to treat other structures as constructed, should we so wish.

${ }^{8}$ The only exceptions I can imagine are the cardinal structures, but again the concession is not too large. I hold that to have a finite cardinality is to have a one-one relationship with a realistic finite ordinal structure, and to have a transfinite cardinality is to have a one-one relationship with real transfinite sets. I believe that this is how we come to understand cardinality in any case, but it would take me too far afield to discuss the epistemic priority of ordinals versus cardinals.

${ }^{9}$ For an excellent account of these problems, see Shapiro 1997, 85-9.
} 
tomorphism problem, realistic structuralists would have postulated the problematic structure itself as realistic.

Hybrid structuralism can therefore accommodate all mathematical structures, without either appealing to special modal intuitions, or countenancing metaphysically dubious indistinguishables. The distinction between basic and constructed structures therefore has much to recommend it, so long as it is introduced at a much lower level than either Parsons or Keränen considered 10

\section{Gonville \&S Caius College}

Trinity Street, Cambridge CB2 1TA, UK

button@cantab.net

\section{References}

Black, Max (1952). 'The identity of indiscernibles'. Mind, 61, pp. 153-64.

Burgess, J (1999). 'Review of Shapiro 1997'. Notre Dame Journal of Formal Logic, 40, pp. 293-91.

Keränen, Jukka (2001). 'The identity problem for realist structuralism'. Philosophia Mathematica, 9, pp. 308-30.

LADYMAn, James (2005). 'Mathematical structuralism and the Identity of Indiscernibles'. Analysis, 65, pp. 218-21.

MacBride, Fraser, editor (Forthcoming). Identity and Modality. Oxford: Oxford University Press.

Parsons, Charles (2004). 'Structuralism and metaphysics'. Philosophical Quarterly, 54, pp. 56-77.

Shapiro, Stewart (1997). Philosophy of Mathematics: Structure and Ontology. New York: Oxford University Press.

Shapiro, Stewart (MSa). 'The governance of identity'. In MACBRIDE (Forthcoming),

ShapIRo, Stewart (MSb). 'Structure and identity'. In MACBRIDE (Forthcoming)

\footnotetext{
${ }^{10}$ Thanks to Alex Paseau, Arif Ahmed and Brian King for helpful discussions concerning identity and structuralism, and for comments on many versions of this paper. Thanks also to Stewart Shapiro, who responded to an earlier draft of this paper at the second Arché Graduate Conference (February 2005) and suggested (B) as a simplification of a formula I had proposed. (At that conference, I approached the Automorphism Problem in a remarkably similar manner to Ladyman 2005, published later that year.)
} 